\title{
Hipoacusia en un paciente tras administración de morfina intratecal
}

\author{
I. Pingarrón, R. Bartol, L.M. Vaquero, F. Sánchez Montero y C. Muriel \\ Servicio de Anestesiología, Reanimación y Terapéutica del Dolor. Hospital Clínico Universitario de \\ Salamanca. Salamanca
}

Pingarrón I, Bartol R, Vaquero LM, Sánchez Montero F, Muriel C. Hipoacusia en un paciente tras administración de morfina intratecal. Rev Soc Esp Dolor 2014; 21(2): 89-91.

\begin{abstract}
The administration of intrathecal opioids is a common technique when treating patients with chronic pain. The chosen drug is morphine due to its pharmacokinetic characteristics that determine a good analgesic quality, still required monitoring of patients for the first 24 hours because of their fundamental risk of respiratory depression.

We present a case of hearing loss associated with opioid intoxication after administration of morphine by intrathecal infusion pump in a patient with chronic pain with post-laminectomy syndrome. The patient was treated with respiratory support and continuous intravenous infusion of naloxone. The hearing loss reverted within one hour after gradual increase of the treatment. The different articles that we analyzed include nausea, vomiting, pruritus, urinary retention and respiratory depression as side effects of intrathecal morphine. There is no reference to the onset of hearing loss associated with intrathecal opioid intoxicaton in the literature we have reviewed so far.
\end{abstract}

Key words: Intrathecal morphine. Hearing loss. Intoxication with opioids.

\section{RESUMEN}

La administración de opioides intradurales es una técnica habitual en el tratamiento de pacientes con dolor crónico. El

Recibido: 15-02-13

Aceptado: 01-05-13. fármaco de elección es la morfina por sus características farmacocinéticas que condicionan una buena calidad analgésica, siendo necesaria la vigilancia de los pacientes durante las primeras 24 horas por el riesgo fundamental de depresión respiratoria.

Presentamos un caso de hipoacusia asociada a la intoxicación por opioides tras la administración de morfina mediante bomba de infusión intratecal en una paciente con dolor crónico por síndrome poslaminectomía. La paciente fue tratada con medidas de soporte respiratorio y perfusión continua intravenosa de naloxona. La hipoacusia revirtió en 1 hora tras incremento progresivo del tratamiento. Los artículos analizados incluyen náuseas, vómitos, prurito, retención urinaria y depresión respiratoria como efectos secundarios de la morfina intratecal. En la literatura médica revisada no hay hasta la fecha ninguna referencia a la aparición de hipoacusia asociada a intoxicación por opioides intradurales.

Palabras clave: Morfina intratecal. Hipoacusia. Intoxicación por opioides.

\section{INTRODUCCIÓN}

Desde hace años, la implantación de bombas de infusión continua de opioides es una alternativa terapéutica en el tratamiento de los pacientes que presentan dolor crónico, ya sea oncológico o no oncológico (1).

La morfina es probablemente el opioide con mayor actividad selectiva medular tras su administración epidural o intradural (2-4). A pesar de los posibles efectos secundarios de este fármaco, el beneficio terapéutico obtenido en estos pacientes es superior. La complicación más temida de la administración intratecal de morfina es la depresión respiratoria. Aparece en las primeras 24 horas, con una incidencia del 0,03-7\%(5-7). 
Hasta la fecha, no existen artículos que relacionen la aparición de hipoacusia con la intoxicación por morfina. Sin embargo, aunque limitados, existen algunos casos de sordera neurosensorial descritos tras el consumo de opioides como heroína, metadona e hidrocodona (8-13).

Presentamos el caso de una paciente con un cuadro de hipoacusia como efecto secundario tras la intoxicación con morfina intratecal. El cuadro revirtió tras la administración de naloxona.

\section{CASO CLÍNICO}

Mujer de 55 años con antecedentes de síndrome poslaminectomía tras una cirugía de discectomía realizada hace 8 años. Tras el fracaso de la terapéutica inicial vía oral decidimos implantar una bomba de infusión intratecal de morfina tipo Medtronic Synchromed ${ }^{\circledR}$ II con reservorio de $40 \mathrm{ml}$ y catéter de $0,14 \mathrm{ml}$. El tratamiento fue efectivo durante 3 años, tras los cuales el alivio sintomático fue progresivamente menor hasta resultar nulo en los últimos 6 meses. Realizamos varios incrementos en la dosis diaria de morfina y la paciente no presentó mejoría. Ante la sospecha de migración del catéter realizamos un estudio radiológico cuyo resultado confirmó el diagnóstico. Ante esta situación decidimos la recolocación del catéter intradural.

La paciente es intervenida bajo anestesia local y sedación, procediendo al recambio de catéter y la posterior conexión a la bomba subcutánea de localización abdominal.

La dosis diaria previa de mórfico intradural para terapia del dolor era de $4 \mathrm{mg} /$ día. Ante la sospecha de que la paciente no recibiese esa dosis desde hace meses, decidimos reiniciar la terapia con una dosis bolo de $1,4 \mathrm{mg}$, que fue administrado durante 2 horas, y $1 \mathrm{mg} /$ día de mantenimiento.

A las 10 horas del inicio de la infusión la paciente comienza con un cuadro de náuseas y vómitos acompañados de síndrome vertiginoso. Realizamos tratamiento con ondasetrón y sulpirida, apreciándose mejoría del cuadro. A las 22 horas la paciente presenta depresión respiratoria con pérdida de consciencia, desaturación y acidosis respiratoria. Iniciamos tratamiento con naloxona $0,8 \mathrm{mg}$ en bolo y ventilación asistida. La enferma recupera la respiración espontánea, aunque permanece obnubilada, y se decide su traslado a la Unidad de Reanimación para continuar tratamiento y vigilancia. Iniciamos la administración de oxigenoterapia y perfusión intravenosa continua de naloxona a dosis de $2 \mathrm{mg} / \mathrm{h}$. La exploración radiológica confirma la correcta posición del catéter. Procedemos al vaciado de la bomba, sustituyendo la perfusión de morfina por suero salino fisiológico. Al poder comunicarnos con ella, refiere no oírnos y a la exploración objetivamos hipoacusia bilateral. Las constantes son: FC 85 lpm, TA 95/45 mm Hg y temperatura de $37^{\circ} \mathrm{C}$. No presenta clínica neurológica y la exploración por aparatos es normal.
Solicitamos interconsulta al servicio de otorrinolaringología. Tras la realización de exploración exhaustiva es diagnosticada de hipoacusia neurosensorial, no encontrando justificación para el cuadro. Tras un periodo de observación de 1 hora con incremento progresivo de naloxona hasta dosis de $6 \mathrm{mg} / \mathrm{h}$, recupera de forma paulatina la audición. Decidimos mantener a la paciente en la unidad de reanimación para observación durante un periodo de 24 horas. Mantenemos el tratamiento antes descrito por el riesgo de un nuevo cuadro de depresión respiratoria. La paciente es dada de alta sin déficit auditivo residual, diagnosticándola de hipoacusia por intoxicación por morfina.

\section{DISCUSIÓN}

Desde 1979 la morfina ha sido utilizada como tratamiento del dolor tanto agudo como crónico. Varias son las características que preconizan su uso por vía raquídea.

La biodisponibilidad sobre los receptores opioides medulares de la morfina, localizados en la sustancia gris de la médula espinal, supera a la del fentanilo y el sulfentanilo lo que la convierte en el opioide con mayor efecto analgésico espinal $(14,15)$.

Los opioides hidrofílicos migran de una manera más rápida y en una extensión mayor que los lipofílicos. Esto podría ser explicado por los diferentes porcentajes de aclaramiento de los distintos opioides en el LCR $(14,16)$. En el caso de la morfina, su aclaramiento es muy bajo con respecto al resto de opioides $(2,8 \mu \mathrm{g} / \mathrm{kg} / \mathrm{min})$, lo que le permite mantenerse durante más tiempo en el LCR. Esto conlleva una mayor posibilidad de difusión rostral, de duración de la analgesia y, consecuentemente, de provocar efectos secundarios supraespinales como sedación y depresión respiratoria.

En nuestro caso, la hipoacusia que la paciente manifiesta al mejorar su estado de consciencia es de difícil explicación.

Podría estar relacionada con la técnica raquídea, pero la hipoacusia en los casos descritos en la bibliografía aparece a las $24-48 \mathrm{~h}$ y suele ceder tras el tratamiento con fluidoterapia o parche hemático (17-19). En este caso la fisiopatología es explicada por la aparición de un hidrops endolinfático por alteraciones de presión en el LCR tras una punción dural.

En nuestro caso el cuadro remitió espontáneamente tras la perfusión de naloxona y la paciente recuperó el $100 \%$ de la capacidad auditiva.

Tras una revisión bibliográfica a través de la base de datos PubMed utilizando los descriptores "morfina intratecal", "efectos adversos", "hipoacusia" y "dolor crónico", no hemos encontrado ninguna referencia bibliográfica respecto a la aparición de hipoacusia asociada a la intoxicación por morfina intradural y vía sistémica. Hay descritos tres casos de hipoacusia en relación con la intoxicación por heroína en pacientes adictos a drogas por vía parenteral (8-10). 
En todos ellos, como en el caso de nuestra paciente, la hipoacusia se asoció a un consumo de opioide tras un tiempo de abstinencia.

Dadas las similitudes con estos casos, la etiología más probable puede ser la intoxicación con morfina intradural.

El mecanismo fisiopatológico podría ser explicado porque algunos neurotransmisores, incluidos lo opioides, participan en la inervación de la cóclea. Se han descrito receptores $\mu, \delta, \kappa(20)$. Eybalin y cols. (21) describieron que tras el tratamiento con agonistas $\mu$ y $\delta$ se producía una inhibición de la actividad basal de la adenilato ciclasa de la cóclea. Este efecto revertía tras la administración de antagonistas opioides (22).

Ishiyama y cols. (8) suponen que la sordera que aparece en los toxicómanos que tras un periodo de abstinencia realizan un consumo ocasional de heroína podría ser un indicativo de una resensibilización de los receptores opioides que previamente habían desarrollado tolerancia (23).

En el caso de nuestra paciente, podríamos concluir que durante el periodo en el que el catéter había migrado no recibió dosis de opioides, por lo tanto se podría considerar un periodo de abstinencia. Tras la conexión del nuevo catéter y reinicio de la terapia con morfina a dosis de $1 \mathrm{mg} /$ día más un bolo de 1,4 mg, sufre una intoxicación por opioides acompañada de hipoacusia. Esta hipoacusia, con los datos aportados, podría ser debida a una hipersensibilización de los receptores opioides $\mu$ cocleares.

Así, podemos concluir que la hipoacusia es un efecto secundario que tener en cuenta y que puede pasar desapercibido en situaciones de intoxicación. Pero son necesarios nuevos casos que puedan ayudar a esclarecer esta hipótesis.

\section{CORRESPONDENCIA:}

Isabel Pingarrón Hernández

Servicio de Anestesiología, Reanimación y Terapéutica del Dolor

Hospital Clínico Universitario de Salamanca

Paseo de San Vicente, 58-182

37007 Salamanca

e-mail: isabelpingarron@gmail.com

\section{BIBLIOGRAFÍA}

1. Wörner J, Kothbauer K, Gerber H. Intrathecal morphine pump malfunction due to leakage at the catheter connection site: A rare problem and its prevention. Anesth Analg 2009;108:1994-5.

2. Gehling M, Tryba M. Risks and side-effects of intrathecal morphine combined with spinal anaesthesia: A meta-analysis. Anaesthesia 2009;64:643-51.

3. Raffaeli W, Marconi G, Fanelli G, Taddei S, Borghi GB, Casati A. Opioid-related side-effects after intrathecal morphine: A prospective, randomized, double-blind dose-response study. Eur J Anaesthesiol 2006;23:605-10.

4. Coffey RJ, Owens ML, Broste SK, Dubois MY, Ferrante FM, Schultz DM, et al. Mortality associated with implantation and management of intrathecal opioid drug infusion systems to treat noncancer pain. Anesthesiology 2009;111:881-91.

5. Rathmell JP, Miller MJ. Death after initiation of intrathecal drug therapy for chronic pain: assessing risk and designing prevention. Anesthesiology 2009;111:706-8.

6. Ouro-Bang'na Maman AF, Sama HD, Alassani F, Egbohou P, Chobli M. Severe differed respiratory depression after intrathecal administration of morphine and clonidine on a 70-year-old patient. Ann Fr Anesth Reanim 2009;28:701-3.

7. Kato R, Shimamoto H, Terui K, Yokota K, Miyao HJ. Delayed respiratory depression associated with $0.15 \mathrm{mg}$ intrathecal morphine for cesarean section: A review of 1915 cases. J Anesth 2008;22:112-6.

8. Ishiyama A, Ishiyama G, Baloh RW, Evans CJ. Heroin-induced reversible profound deafness and vestibular dysfunction. Addiction 2001;96:1363-4.

9. Schrock A, Jakob M, Wirz S, Bootz F. Sudden sensorineural hearing loss after heroin injection. Eur Arch Otorhinolaryngol 2008;265:603-6.

10. Schrock A, Jakob M, Wirz S, Bootz F. Sudden sensorineural hearing loss after heroin inyection. Eur Arch Otorhinolaryngol 2008;265:603-6.

11. Van Gaalen FA, Compier EA, Fogteloo AJ. Sudden hearing loss after a methadone overdose. Eur Arch Otorhinolaryngol 2009;266:773-4.

12. Christenson BJ, Marjala AR. Two cases of sudden sensorineural hearing loss after methadone overdose. Ann Pharmacother 2010;44:207-10.

13. Ho T, Vrabec JT, Burton AW. Hydrocodone use and sensorineural hearing loss. Pain Physician 2007;10:467-72.

14. Mugabure B, Echaniz E, Marin M. Fisiología y farmacología clínica de los opioides intradurales e intratecales. Rev Soc Esp Dolor 2005;1:33-45.

15. Christopher M Bernards. Understanding the physiology and pharmacology of epidural and intrathecal opioids. Best Pract Res Clin Anaesthesiol 2002;16:489-505.

16. Brill S, Gurman GM, Fisher A. A history of neuraxial administration of local analgesics and opioids. Eur J Anaesthesiol 2004;21:329-30.

17. Erol A, Topal A, Arbag H, Kilicaslan A, Reisli R, Otelcioglu S. Auditory function after spinal anaesthesia: The effect of differently designed spinal needles. Eur J Anaesthesiol 2009;26:416-20.

18. Finegold HD, Mandell G, Vallejo M, Ramanathan S. Does spinal anesthesia cause hearing loss in the obstetric population? Anesth Analg 2002;95:198-203.

19. Gültekin S, Ozcan S. Does hearing loss after spinal anesthesia differ between young and elderly patients? Anesth Analg 2002;94:1318-20.

20. Jongkamonwiwat N, Phansuwan-Pujito P, Sarapoke P, Chetsawang B, Casalotti S, Forge A, et al. The presence of opioid receptors in rat inner ear. Hear Res 2003;181:85-93.

21. Eybalin M, Pujol R, Bockaert J. Opioid receptors inhibit the adenylate cyclase in guinea pig cochleas. Brain Res 1987;421: 336-42.

22. Pradhan AA, Siau C, Constantin A, Clarke PB. Chronic morphine administration results in tolerance to delta opioid receptor-mediated antinociception. Neuroscience 2006;141:947-54.

23. Dunbar SA, Karamian IG. Periodic abstinence enhances nociception without significantly altering the antinociceptive efficacy of spinal morphine in the rat. Neurosci Lett 2003;344:145-8. 